\title{
molecules
}

ISSN 1420-3049

Article

www.mdpi.com/journal/molecules

\section{Synthesis and Antifungal Activity of Novel Sulfone Derivatives Containing 1,3,4-Oxadiazole Moieties}

\author{
Weiming Xu *, Jiang He, Ming He, Feifei Han, Xuehai Chen, Zhaoxi Pan, Jian Wang and \\ Maoguo Tong
}

State Key Laboratory Breeding Base of Green Pesticide and Agricultural Bioengineering, Key Laboratory of Green Pesticide and Agricultural Bioengineering, Ministry of Education, Center for Research and Development of Fine Chemicals, Guizhou University, Guiyang 550025, China;

E-Mails: he6701324jiang@163.com (J.H.); hmher@126.com (M.H.); yougubaihe101@163.com (F.H.); chenxuehai2010@foxmail.com(X.C.); panpan0301-121@163.com (Z.P.); wangjmy2008@126.com (J.W.); tongmaoguo@163.com (M.T.)

* Author to whom correspondence should be addressed; E-Mail: xuweiming2009@163.com; Tel.: +86-851-362-0521; Fax: +86-851-362-2211.

Received: 8 October 2011; in revised form: 24 October 2011 / Accepted: 25 October 2011 / Published: 1 November 2011

\begin{abstract}
A series of new sulfone compounds containing 1,3,4-oxadiazole moieties were synthesized. The structures of these compounds were confirmed by spectroscopic data (IR, ${ }^{1} \mathrm{H}$ - and $\left.{ }^{13} \mathrm{C}-\mathrm{NMR}\right)$ and elemental analyses. Antifungal tests indicated that all the title compounds exhibited good antifungal activities against eight kinds of plant pathogenic fungi, and some showed superiority over the commercial fungicide hymexazol. Among them, compounds $\mathbf{5 d}, \mathbf{5 e}, \mathbf{5 f}$, and $\mathbf{5 i}$ showed prominent activity against $B$. cinerea, with determined $\mathrm{EC}_{50}$ values of $5.21 \mu \mathrm{g} / \mathrm{mL}, 8.25 \mu \mathrm{g} / \mathrm{mL}, 8.03 \mu \mathrm{g} / \mathrm{mL}$, and $21.00 \mu \mathrm{g} / \mathrm{mL}$, respectively. The present work demonstrates that sulfone derivatives such as $\mathbf{5 d}$ containing a 1,3,4-oxadiazole moiety can be used as possible lead compounds for the development of potential agrochemicals.
\end{abstract}

Keywords: sulfone; oxadiazole; antifungal activity 


\section{Introduction}

The emergence of fungal resistance to existing fungicides has posed a serious concern for pesticide professionals during the last decade, and the desire for safer and more effective agrochemicals with reduced environmental toxicity also remains a high priority [1]. A further aim is to produce novel fungicides that do not impede the role of beneficial organisms in plant development and which do not persist in the environment and food chains [2], so the synthesis and antifungal evaluation of new compounds is greatly needed.

In this context, sulfone derivatives provide an example of an important class of bioactive compounds with a wide spectrum of activities, as the sulfone group is an important core found in numerous biologically active compounds with a wide range of biological activity including insecticidal [3], antifungal [4], herbicidal [5], anti-hepatitis [6], antitumor [7], anti-inflammatory [8], anticancer [9], anti-HIV-1 [10] and anti-tubercular [11] properties. There is evidence that the key feature of these compounds is a 5- or 6-membered heterocyclic ring attached to a sulfone, and additional modification of the heterocyclic ring has been considered. Among these derivatives, a 2-((4-chlorobenzyl)sulfonyl)5-(methylsulfonyl)-1,3,4-thiadiazole sulfone prepared by Joachim et al. exhibited good inhibitory activity against Plasmopara viticola [12] at a concentration of $1 \times 10^{-5} \mathrm{mg} / \mathrm{kg}, 2$,4-dibromo-5-methyl-1((2-methyl-5-nitrophenyl)sulfonyl)- $1 H$-imidazole sulfone, reported by Assmann et al., exhibited strong activity against Phytophthora infestans and Plasmopara viticola [13] at a concentration of $50 \mathrm{~g} / \mathrm{ha}$, and 2-(5-ethyl-1-methyl-1H-pyrazol-3-yl)-5-(methylsulfonyl)-1,3,4-oxadiazole sulfone, prepared by Yuan et al., exhibited medium inhibitory activity against Phoma asparagi [14]. As an illustration of a practical application, the agricultural fungicide oxycarboxin was successfully commercialized by Uniroyal Co. in the year 1966. In the past few decades, a large number of other fungicides with potent bioactivity containing sulfone units such as tolylfluanid, dichlofluanid, cyazofamid, amisulbrom and oryzaemate have been introduced in the market by various companies $[15,16]$.

The 1,3,4-oxadiazole scaffold is an important pharmacophore in agricultural science and compounds bearing this moiety often display antifungal [17], herbicidal [18] and insecticidal [19,20] activities. As a illustration of the activity of 1,3,4-oxadiazole sulfones, Keshari et al. reported that 2-(5sulfanyl-1,3,4-oxadiazol-2-yl)phenylacetate and 5-(pyridin-3-yl)-1,3,4-oxadiazole-2-thiol exhibit good antibacterial activities against Escherichia coli (MTCC 443) [21].

As part of our ongoing search for novel sulfone compounds possessing antifungal properties, new derivatives of 2-sulfonyl-5-(3,4,5-trimethoxyphenyl)-1,3,4-oxadiazole (I) [22] and 2-sulfinyl-5-(3,4,5trimethoxyphenyl)-1,3,4-oxadiazole (II) [23] were synthesized. Subsequent in vitro bioassays disclosed that the compounds 2-(methylsulfonyl)-5-(3,4,5-trimethoxyphenyl)-1,3,4-oxadiazole (Ia) and 2-(benzylsulfinyl)-5-(3,4,5-trimethoxyphenyl)-1,3,4-oxadiazole (IIa) possess high antifungal activities against 10 kinds of fungi, with $\mathrm{EC}_{50}$ values ranging from $19.9 \mu \mathrm{g} / \mathrm{mL}$ to $93.3 \mu \mathrm{g} / \mathrm{mL}$, being equivalent or more potent against the tested fungi than the commercial agricultural fungicide hymexazol, and they also showed broad-spectrum bioactivity.

The SAR suggested that 2-(methylsulfonyl)-1,3,4-oxadiazole is the core ring system that affords potent antifungal activities [22,23]. Given the apparent lack of scope for changes to the core ring system, the majority of our efforts were directed towards compounds of the general structure of the title compounds in which the core ring system was kept constant and the peripheral groups were 
altered. As a consequence, in an attempt to increase the fitting to the pharmacophoric model, and possibly to obtain new fungicides, we report here the synthesis, characterization and antifungal activity of novel sulfone derivatives containing 1,3,4-oxadiazole moieties 5, as shown in Scheme 1.

Scheme 1. Synthetic route to the title compounds.

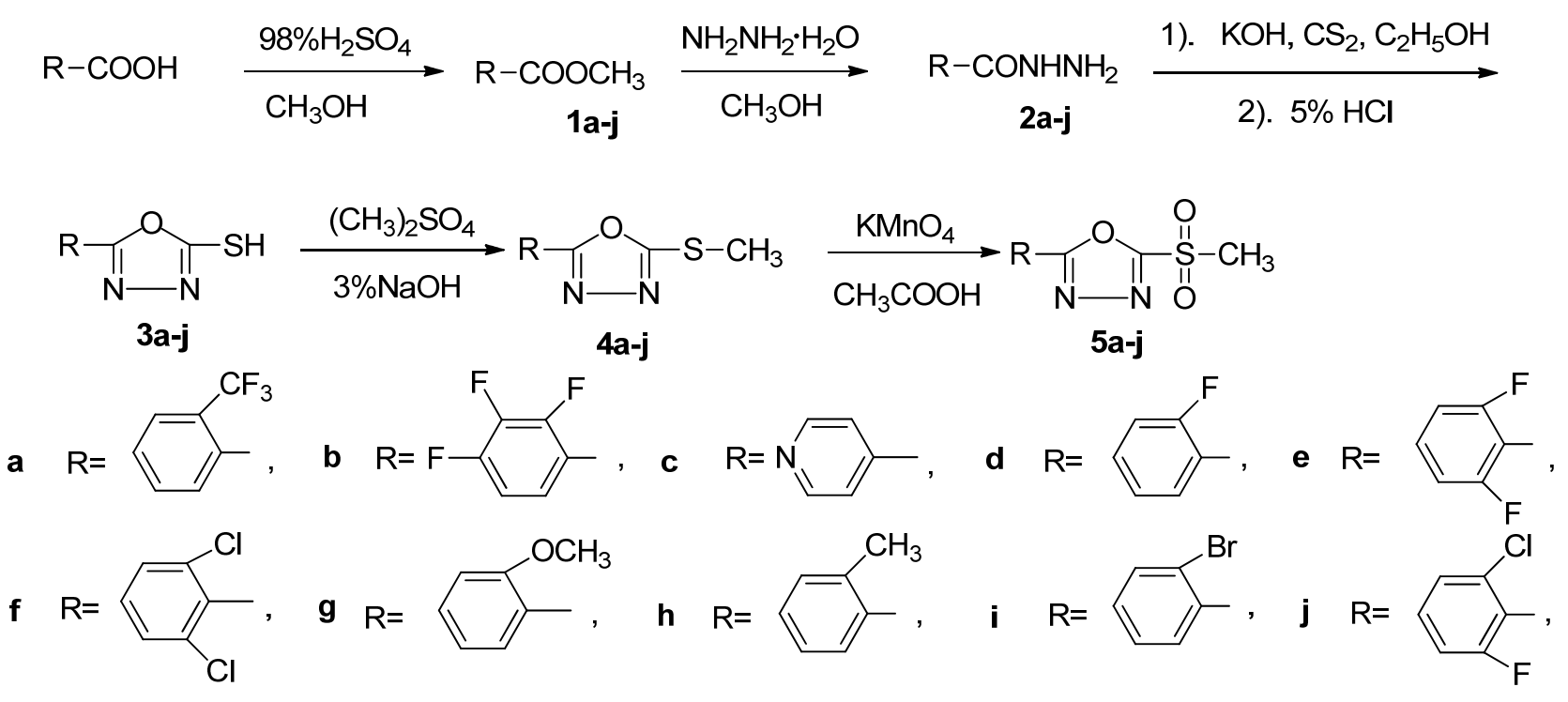

\section{Results and Discussion}

\subsection{Chemistry Benzohydrazide}

The key intermediate 2-thiol-5-substituted-1,3,4-oxadiazole (3) was prepared by cyclization of substituted phenylhydrazide, potassium hydroxide, and carbon disulfide in ethanol under reflux conditions. The key point of this reaction is that water must be removed completely; as the presence of even a little water may cause the cyclization to fail.

Although the electron rich methylthio moiety in compounds 4 can be oxidized to a sulfone by a variety of agents such as $m$-CPBA [24] or $\mathrm{H}_{2} \mathrm{O}_{2}$ catalyzed by methyltrioxorhenium [25], unfortunately, most of these reagents are not satisfactory. They are either harmful or expensive, and a simple procedure is not easily available. In this experiment, the methylthio moiety in $\mathbf{4}$ was oxidized with potassium permanganate [26] in glacial acetic acid to afford the corresponding methylsulfonyl species $\mathbf{5}$, the advantage of this oxidation is that it uses an inexpensive oxidant without a catalyst; furthermore, the reaction is quickly complete and easy to work up. The physical characteristics, IR, ${ }^{1} \mathrm{H}-\mathrm{NMR}$, ${ }^{13} \mathrm{C}-\mathrm{NMR}$ and elemental analyses data for all the synthesized compounds are reported in the Experimental section.

\subsection{The Antifungal Activities of Oxadiazole Methyl Sulfones}

The inhibitory effects of the synthesized oxadiazole methyl sulfone compounds on phytopathogenic fungi was studied. Two fungi, $F$. oxysporum and $C$. mandshurica, representing typical fungi often occurring in the Chinese agro-ecosystem were chosen for fungicide screening using the mycelial growth rate method. The results were compared with that of the commercial agricultural fungicide hymexazol (a broad spectrum fungicide), as indicated in Table 1. 
Table 1. Inhibition effect of oxadiazole methyl sulfones against phytopathogenic fungi at $50 \mu \mathrm{g} / \mathrm{mL}$.

\begin{tabular}{|c|c|c|c|}
\hline \multirow{2}{*}{ Compound } & \multirow{2}{*}{$\mathbf{R}$} & \multicolumn{2}{|c|}{ Inhibition (\%) } \\
\hline & & F. oxysporum & C. mandshurica \\
\hline $5 \mathbf{a}$ & & $71.1 \pm 7.7$ & $68.3 \pm 9.3$ \\
\hline $5 \mathbf{b}$ & & $97.5 \pm 3.3$ & $89.7 \pm 3.1$ \\
\hline $5 c$ & & $72.6 \pm 6.4$ & $78.2 \pm 4.7$ \\
\hline $5 d$ & & $70.1 \pm 4.9$ & $64.0 \pm 1.3$ \\
\hline $5 e$ & & $98.8 \pm 8.0$ & $97.8 \pm 11.8$ \\
\hline $5 f$ & & $94.0 \pm 4.1$ & $97.6 \pm 4.3$ \\
\hline $5 g$ & & $89.6 \pm 4.5$ & $91.3 \pm 9.6$ \\
\hline $5 \mathrm{~h}$ & & $74.6 \pm 8.5$ & $99.3 \pm 12.8$ \\
\hline $5 \mathbf{i}$ & & $77.0 \pm 6.6$ & $79.6 \pm 7.1$ \\
\hline $5 \mathbf{j}$ & & $67.1 \pm 4.8$ & $64.0 \pm 2.6$ \\
\hline & Hymexazol & $58.4 \pm 0.8$ & $57.3 \pm 0.3$ \\
\hline
\end{tabular}

As indicated in Table 1, at the concentration of $50 \mu \mathrm{g} / \mathrm{mL}$, all of the tested compounds exhibited good inhibitory effects against $F$. oxysporum, and all of them showed superiority over the commercial fungicide hymexazol. Among them, compounds $\mathbf{5 b}, \mathbf{5 e}$ and $\mathbf{5 f}$ almost completely inhibited the growth of F. oxysporum, with control efficacies of $97.5 \%, 98.8 \%$, and $94.0 \%$, respectively. Compound $\mathbf{5 g}$ showed $89.6 \%$ fungicidal activity against $F$. oxysporum, while the others had inhibition activities between $67.1 \%$ and $77.0 \%$. As the results described in Table 1 indicate, all of the tested compounds possessed promising inhibitory effects against C. mandshurica. Among them, compounds 5e, 5f, 5g, 
and $\mathbf{5 h}$ almost completely inhibited the growth of $C$. mandshurica, with inhibition values of more than 90.0\%, whereas compound 5b showed $89.7 \%$ fungicidal activity against $C$. mandshurica, and compounds $\mathbf{5 a}, \mathbf{5 c}, \mathbf{5 d}, \mathbf{5 i}$, and $\mathbf{5 j}$ inhibited the growth of $C$. mandshurica by $68.3 \%, 78.2 \%, 64.0 \%$, $79.6 \%$, and $64.0 \%$, respectively.

\subsection{Toxicity of Some Title Compounds on 8 Kinds of Pathogenic Fungi}

We choose some representative compounds with good, ordinary antifungal activity, as indicated in the previous bioassays, to conduct further work which disclosed that some sulfone compounds containing a methyl oxadiazole showed remarkable inhibitory effect on eight kinds of plant pathogenic fungi, which represent typical fungi often occurring in the Chinese agro-ecosystem. The results are summarized in Table 2.

Table 2. Toxicity of some methyl sulfones on eight kinds of pathogenic fungi.

\begin{tabular}{|c|c|c|c|c|}
\hline Compounds & Fungi & Toxic regression equation & $\mathrm{EC}_{50}(\mu \mathrm{g} / \mathrm{mL})$ & $\mathbf{R}$ \\
\hline \multirow{8}{*}{$5 d$} & C. mandshurica & $y=1.428 x+2.283$ & $79.92 \pm 14.79$ & 0.861 \\
\hline & F. oxysporum & $y=1.355 x+3.017$ & $29.07 \pm 7.82$ & 0.952 \\
\hline & R. solani & $y=2.163 x+3.251$ & $6.43 \pm 1.34$ & 0.878 \\
\hline & B. cinerea & $y=1.341 x+4.038$ & $5.21 \pm 2.05$ & 0.921 \\
\hline & P. infestans & $y=1.372 x+3.397$ & $14.73 \pm 3.23$ & 0.846 \\
\hline & C. gloeosporioides & $\mathrm{y}=2.930 \mathrm{x}+1.173$ & $20.23 \pm 6.65$ & 0.961 \\
\hline & S. sclerotiorum & $y=1.860 x+3.272$ & $8.49 \pm 3.51$ & 0.919 \\
\hline & T. cucumeris & $y=3.537 x+1.460$ & $10.01 \pm 5.64$ & 0.974 \\
\hline \multirow{8}{*}{$5 e$} & C. mandshurica & $y=3.623 x-0.735$ & $38.27 \pm 3.21$ & 0.867 \\
\hline & F. oxysporum & $y=1.439 x+2.384$ & $65.75 \pm 7.04$ & 0.976 \\
\hline & T. cucumeris & $y=7.95 x-5.878$ & $23.35 \pm 4.76$ & 0.980 \\
\hline & R. solani & $y=3.681 x+0.115$ & $21.23 \pm 4.12$ & 0.916 \\
\hline & B. cinerea & $y=1.993 x+3.173$ & $8.25 \pm 0.85$ & 0.853 \\
\hline & P. infestans & $y=1.216 x+2.842$ & $59.52 \pm 16.79$ & 0.991 \\
\hline & C. gloeosporioides & $y=4.629 x-1.556$ & $26.07 \pm 7.32$ & 0.943 \\
\hline & S. sclerotiorum & $y=5.984 x-2.034$ & $14.97 \pm 6.83$ & 0.974 \\
\hline \multirow{8}{*}{$5 f$} & C. mandshurica & $y=1.131 x+2.747$ & $98.18 \pm 8.35$ & 0.981 \\
\hline & F. oxysporum & $y=1.081 x+2.912$ & $85.41 \pm 17.92$ & 0.988 \\
\hline & T. cucumeris & $y=2.381 x+1.661$ & $25.25 \pm 2.34$ & 0.911 \\
\hline & R. solani & $y=2.432 x+2.061$ & $16.16 \pm 9.76$ & 0.916 \\
\hline & B. cinerea & $y=2.528 x+2.712$ & $8.03 \pm 0.86$ & 0.962 \\
\hline & P. infestans & $y=1.163 x+3.101$ & $42.93 \pm 7.38$ & 0.993 \\
\hline & C. gloeosporioides & $y=1.861 x+2.171$ & $33.12 \pm 8.29$ & 0.979 \\
\hline & S. sclerotiorum & $y=5.036 x-1.223$ & $17.20 \pm 4.72$ & 0.951 \\
\hline \multirow{8}{*}{$5 \mathbf{i}$} & F. oxysporum & $y=4.243 x-1.261$ & $29.89 \pm 1.31$ & 0.918 \\
\hline & C. mandshurica & $y=4.355 x-2.179$ & $44.50 \pm 3.56$ & 0.947 \\
\hline & R. solani & $y=5.036 x-1.879$ & $20.02 \pm 1.28$ & 0.978 \\
\hline & T. cucumeris & $y=5.285 x-3.994$ & $24.78 \pm 4.29$ & 0.964 \\
\hline & S. sclerotiorum & $y=2.562 x+2.003$ & $14.78 \pm 1.02$ & 0.879 \\
\hline & B. cinerea & $y=7.582 x-5.026$ & $21.00 \pm 2.01$ & 0.947 \\
\hline & C. gloeosporioides & $y=6.364 x-3.537$ & $21.95 \pm 2.93$ & 0.963 \\
\hline & P. infestans & $y=1.358 x+2.697$ & $49.64 \pm 9.39$ & 0.958 \\
\hline
\end{tabular}


Table 2. Cont.

\begin{tabular}{llllc}
\hline Compounds & \multicolumn{1}{c}{ Fungi } & Toxic regression equation & $\mathbf{E C}_{\mathbf{5 0}}(\boldsymbol{\mu g} / \mathbf{m L})$ & $\mathbf{R}$ \\
\hline & F. oxysporum & $\mathrm{y}=1.343 \mathrm{x}+3.058$ & $27.93 \pm 1.02$ & 0.980 \\
& C. mandshurica & $\mathrm{y}=2.103 \mathrm{x}+1.647$ & $39.26 \pm 2.79$ & 0.999 \\
& R. solani & $\mathrm{y}=3.532 \mathrm{x}-0.604$ & $38.64 \pm 0.45$ & 0.880 \\
Hymexazol & T. cucumeris & $\mathrm{y}=1.298 \mathrm{x}+3.043$ & $32.21 \pm 5.82$ & 0.958 \\
& S. sclerotiorum & $\mathrm{y}=2.346 \mathrm{x}+2.900$ & $7.76 \pm 2.98$ & 0.998 \\
& C. gloeosporioides & $\mathrm{y}=3.896 \mathrm{x}-1.136$ & $37.58 \pm 3.16$ & 0.946 \\
& P. infestans & $\mathrm{y}=1.715 \mathrm{x}+2.559$ & $26.49 \pm 1.42$ & 0.858 \\
& B. cinerea & $\mathrm{y}=2.014 \mathrm{x}+2.177$ & $25.23 \pm 6.12$ & 0.917 \\
\hline
\end{tabular}

As indicated in Table 2, all of the test compounds possessed prominent antifungal activities against eight plant pathogens (F. oxysporum, C. mandshurica, R. solani, T. cucumeris, S. sclerotiorum, C. gloeosporioides, $P$. infestans, B. cinerea), with $\mathrm{EC}_{50}$ values between $5.21 \mu \mathrm{g} / \mathrm{mL}$ to $98.18 \mu \mathrm{g} / \mathrm{mL}$. Among them, the bioassay results showed that compounds $5 \mathbf{d}$, 5e, 5f, and $5 \mathbf{i}$ showed prominent activity against $R$. solani $\left(\mathrm{EC}_{50}\right.$ values of $6.43 \mu \mathrm{g} / \mathrm{mL}, 21.23 \mu \mathrm{g} / \mathrm{mL}, 16.16 \mu \mathrm{g} / \mathrm{mL}$, and $20.02 \mu \mathrm{g} / \mathrm{mL}$, respectively), which was superior to the activity of the commercial fungicide hymexazol $(38.64 \mu \mathrm{g} / \mathrm{mL})$. B. cinerea has been identified as a pathogen of more than 235 plant species, including grapes, lettuce, tomatoes, tobacco, and strawberries, producing a gray powdery mold on the infected crops. To our delight, compounds $\mathbf{5 d}, \mathbf{5 e}, \mathbf{5 f}$, and $\mathbf{5 i}$ showed prominent activity against $B$. cinerea, the $\mathrm{EC}_{50}$ values of $5.21 \mu \mathrm{g} / \mathrm{mL}, 8.25 \mu \mathrm{g} / \mathrm{mL}, 8.03 \mu \mathrm{g} / \mathrm{mL}$, and $21.00 \mu \mathrm{g} / \mathrm{mL}$, respectively, all of them being more effective than the positive control. The results also suggested that compound $\mathbf{5 d}$ can be used as a possible lead compound for the development of potential agrochemicals. Compounds $\mathbf{5 d}$, 5e, $\mathbf{5 f}$, and $\mathbf{5 i}$ showed ordinary activity against $S$. sclerotiorum, the $\mathrm{EC}_{50}$ values of $8.49 \mu \mathrm{g} / \mathrm{mL}, 14.97 \mu \mathrm{g} / \mathrm{mL}, 17.20 \mu \mathrm{g} / \mathrm{mL}$, and $14.78 \mu \mathrm{g} / \mathrm{mL}$, respectively, less effective than the positive control hymexazol $(7.76 \mu \mathrm{g} / \mathrm{mL})$. Compounds $\mathbf{5 d}$, 5e, 5f, and $\mathbf{5 i}$ showed weak effectivity against $C$. mandshurica, with $\mathrm{EC}_{50}$ values of $79.92 \mu \mathrm{g} / \mathrm{mL}, 38.27 \mu \mathrm{g} / \mathrm{mL}, 98.18 \mu \mathrm{g} / \mathrm{mL}$, and $44.50 \mu \mathrm{g} / \mathrm{mL}$, respectively. Generally speaking, compounds 5d, 5e, 5f, and $\mathbf{5 i}$ showed weak to normal effective against $F$. oxysporum, $T$. cucumeris, S. sclerotiorum, C. gloeosporioides, and P. infestans, with $\mathrm{EC}_{50}$ values ranging from $14.73 \mu \mathrm{g} / \mathrm{mL}$ to $85.41 \mu \mathrm{g} / \mathrm{mL}$. As indicated in Table 1 and Table 2, with C. mandshurica as the experimental target the relationship between antifungal activity and substituent attached to the benzene ring showed that a fluorinated phenyl group, such as compound 5e $(\mathrm{R}=2,6-2 \mathrm{~F})$, had better antifungal activity $(97.8 \%$ fungicidal activity against $C$. mandshurica at $50 \mu \mathrm{g} / \mathrm{mL}$, and the $\mathrm{EC}_{50}$ was $38.27 \mu \mathrm{g} / \mathrm{mL}$ ).

Compound 5d had prominent antifungal activities against most of the tested fungi, and showed a broad-spectrum bioactivity; the inhibition effects of compound 5d on mycelia growth in vitro at different concentrations are illustrated in Figure 1. 
Figure 1. Effect of different concentrations of $\mathbf{5 d}$ on the mycelial growth of pathogenic fungi $(50,25,12.5,6.25,3.125,0 \mu \mathrm{g} / \mathrm{mL}$, the smaller of zone, the higher of concentration).

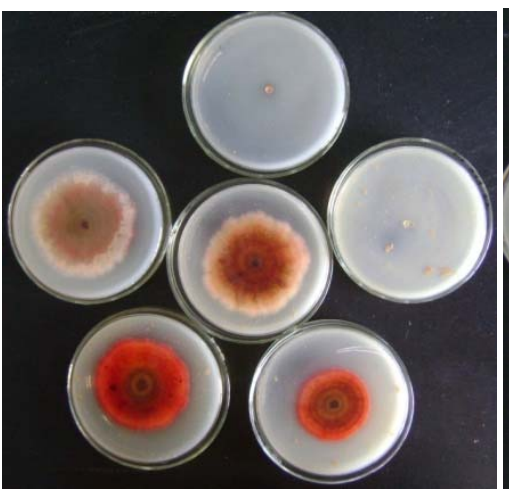

R. solani

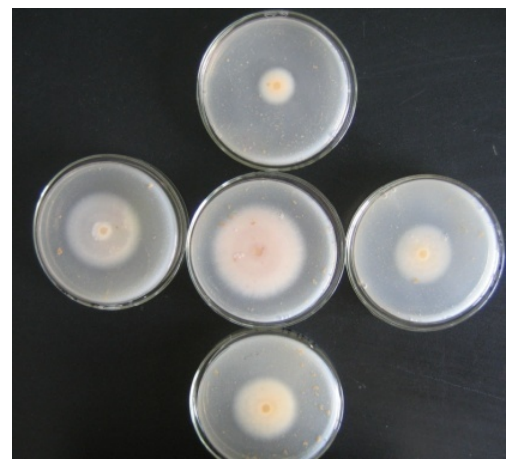

F. oxysporum

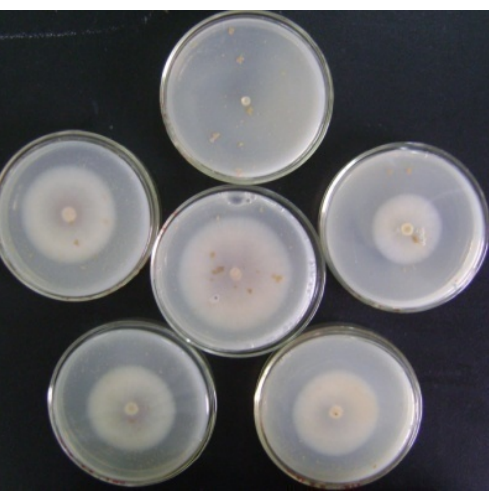

P. infestans

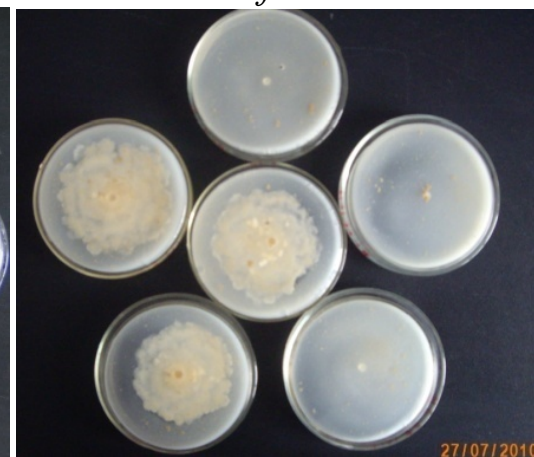

S. sclerotiorum

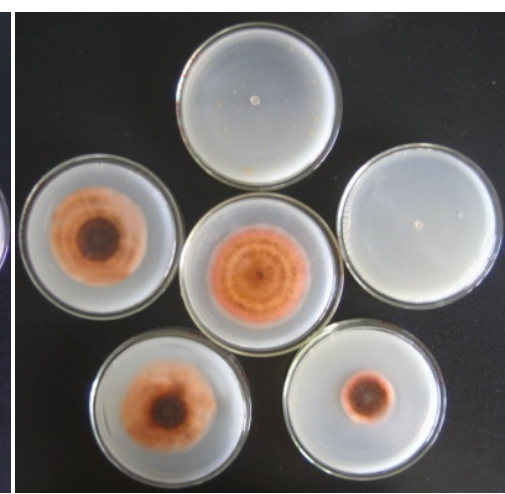

T. cucumeris

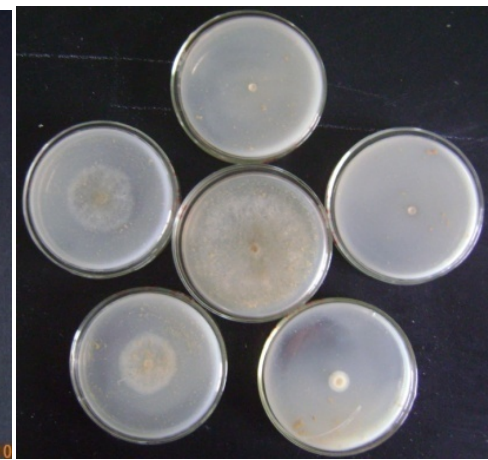

B. Cinerea

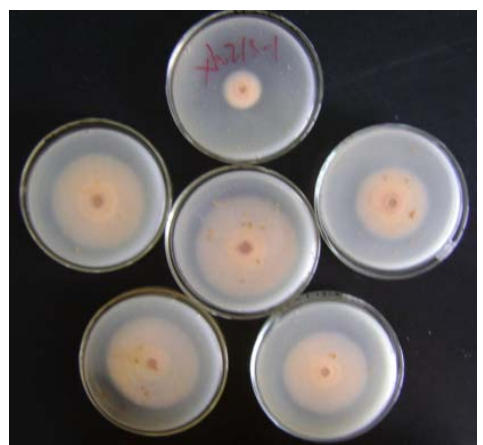

C. mandshurica

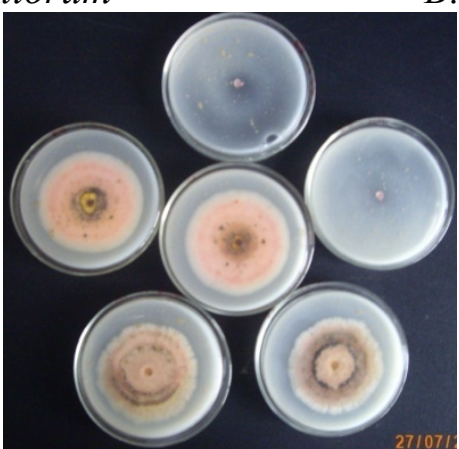

C. gloeosporioides

\section{Experimental}

\subsection{General}

Unless otherwise indicated, all common reagents and solvents were used as obtained from commercial suppliers without further purifications. The melting points of the products were determined on a XT-4 binocular microscope (Beijing Tech Instrument Co., China) and were not corrected. The IR spectra were recorded on a Bruker VECTOR 22 spectrometer in $\mathrm{KBr}$ disks. ${ }^{1} \mathrm{H}-$ and ${ }^{13} \mathrm{C}$-NMR spectra (solvent $\mathrm{CDCl}_{3}$ ) were recorded on a JEOL-ECX 500 NMR spectrometer at room temperature using TMS as an internal standard, chemical shift values $(\delta)$ are given in parts per million. Elemental analysis was performed on an Elementar Vario-III CHN analyzer. Analytical TLC was performed on silica gel GF254. Column chromatographic purification was carried out using silica gel. 


\subsection{Preparation of the Intermediates 1-3}

Starting from the corresponding acid, and following the reported method $[27,28]$, intermediates 3 were synthesized in three steps involving esterification, hydrazination, and cyclization. Specifically, 2-thiol-5-substituted-1,3,4-oxadiazole intermediates 3 were prepared by the reaction of substituted phenylhydrazide intermediates $\mathbf{2}$, potassium hydroxide, and carbon disulfide in ethanol under reflux conditions. Intermediates $\mathbf{2}$ were synthesized from intermediates $\mathbf{1}$ and hydrazine hydrate in methanol under reflux condition. Intermediates $\mathbf{1}$ (substituted benzoic acid esters) were made through esterification reactions from the starting acids.

\subsection{Preparation of the Intermediates $\mathbf{4}$}

To a solution of 2-thiol-5-substituted-1,3,4-oxadiazole $3(2 \mathrm{mmol})$ and sodium hydroxide $(0.08 \mathrm{~g}$, $2 \mathrm{mmol})$ in water $(20 \mathrm{~mL})$, dimethyl sulfate $(0.32 \mathrm{~g}, 2.5 \mathrm{mmol})$ was added dropwise. After stirring for $3 \mathrm{~h}$ at room temperature $\left(20-23{ }^{\circ} \mathrm{C}\right)$, for solid products, the precipitate was filtered off, washed with $5 \%$ $\mathrm{Na}_{2} \mathrm{CO}_{3}$ solution and distilled water, dried and recrystallized from ethanol to afford the white solid products 4; for liquid products, the reaction mixture was washed with $5 \% \mathrm{Na}_{2} \mathrm{CO}_{3}$ solution, and then extracted with ethyl ether $(4 \times 20 \mathrm{~mL})$, the ethyl ether layer was dried with anhydrous $\mathrm{Na}_{2} \mathrm{SO}_{4}$, after removal of the organic solvent under reduced pressure, and oily products 4 were thus obtained.

2-(Methylthio)-5-(2-(trifluoromethyl)phenyl)-1,3,4-oxadiazole (4a): yield 79.2\%; white solid; m.p. 76-78 ${ }^{\circ} \mathrm{C} ;{ }^{1} \mathrm{H}-\mathrm{NMR} \delta: 7.58-7.43\left(\mathrm{~m}, 4 \mathrm{H}\right.$, benzene-H), 3.15 (s, $\left.3 \mathrm{H}, \mathrm{SCH}_{3}\right) ;{ }^{13} \mathrm{C}-\mathrm{NMR} \delta: 162.23,159.43$, 133.05, 132.78, 132.31, 131.67, 131.03, 122.34, 14.73.

2-(Methylthio)-5-(2,3,4-trifluorophenyl)-1,3,4-oxadiazole (4b): yield 77.9\%; oil; ${ }^{1} \mathrm{H}-\mathrm{NMR} \delta$ : $7.88-7.46$ (m, $2 \mathrm{H}$, benzene-H), $3.22\left(\mathrm{~s}, 3 \mathrm{H}, \mathrm{SCH}_{3}\right)$.

2-(Methylthio)-5-(pyridin-4-yl)-1,3,4-oxadiazole (4c): yield 75.4\%; oil. ${ }^{1} \mathrm{H}-\mathrm{NMR} \delta$ : 8.66-7.47 (m, 4H, pyridin-H), 2.91 (s, $\left.3 \mathrm{H}, \mathrm{SCH}_{3}\right)$.

2-(Methylthio)-5-(2-fluorophenyl)-1,3,4-oxadiazole (4d): yield 85.0\%; oil; ${ }^{1} \mathrm{H}-\mathrm{NMR} \delta: 7.61-7.25$ (m, $4 \mathrm{H}$, benzene-H), $2.93\left(\mathrm{~s}, 3 \mathrm{H}, \mathrm{SCH}_{3}\right) ;{ }^{13} \mathrm{C}-\mathrm{NMR} \delta: 163.41,159.94,159.73,136.37,133.71,132.32$, $129.74,121.45,14.71$.

2-(Methylthio)-5-(2,6-difluorophenyl)-1,3,4-oxadiazole (4e): yield 88.4\%; oil; ${ }^{1} \mathrm{H}-\mathrm{NMR} \delta$ : $7.84-7.27$ (m, 3H, benzene-H), $2.78\left(\mathrm{~s}, 3 \mathrm{H}, \mathrm{SCH}_{3}\right) ;{ }^{13} \mathrm{C}-\mathrm{NMR} \delta: 165.73,164.15,153.63,151.74,149.75,123.50$, $118.38,116.23,14.72$.

2-(Methylthio)-5-(2,6-dichlorophenyl)-1,3,4-oxadiazole (4f): yield 78.3\%; oil; ${ }^{1} \mathrm{H}-\mathrm{NMR} \delta: 7.63-7.24$ (m, 3H, benzene-H), $2.81\left(\mathrm{~s}, 3 \mathrm{H}, \mathrm{SCH}_{3}\right)$.

2-(Methylthio)-5-(2-methoxyphenyl)-1,3,4-oxadiazole (4g): yield 79.0\%; oil; ${ }^{1} \mathrm{H}-\mathrm{NMR} \delta: 7.88-7.27$ (m, $4 \mathrm{H}$, benzene-H), $3.02\left(\mathrm{~s}, 3 \mathrm{H}, \mathrm{OCH}_{3}\right), 2.79\left(\mathrm{~s}, 3 \mathrm{H}, \mathrm{SCH}_{3}\right) .{ }^{13} \mathrm{C}-\mathrm{NMR} \delta: 165.23,162.43,138.69,131.87$, $128.77,125.22,122.37,45.23,14.43$. 
2-(Methylthio)-5-(2-methylphenyl)-1,3,4-oxadiazole (4h): yield 85.2\%; oil; ${ }^{1} \mathrm{H}-\mathrm{NMR} \delta$ : $7.88-7.27$ (m, $4 \mathrm{H}$, benzene-H), $2.79\left(\mathrm{~s}, 3 \mathrm{H}, \mathrm{SCH}_{3}\right), 2.68\left(\mathrm{~s}, 3 \mathrm{H}, \mathrm{CH}_{3}\right) ;{ }^{13} \mathrm{C}-\mathrm{NMR} \delta: 166.14,161.67,138.34,131.92$, $128.80,126.21,121.43,22.13,14.68$.

2-(Methylthio)-5-(2-bromophenyl)-1,3,4-oxadiazole (4i): yield 85.3\%; oil; ${ }^{1} \mathrm{H}-\mathrm{NMR} \delta: 7.81-7.44$ (m, $4 \mathrm{H}$, benzene-H), $3.03\left(\mathrm{~s}, 3 \mathrm{H}, \mathrm{SCH}_{3}\right)$.

2-(Methylthio)-5-(2-chloro-6-fluorophenyl)-1,3,4-oxadiazole (4j): yield 79.3\%; oil; ${ }^{1} \mathrm{H}-\mathrm{NMR} \quad \delta: 7.94-$ 7.33 (m, 3H, benzene-H), 2.71 (s, 3H, $\left.\mathrm{SCH}_{3}\right)$.

\subsection{Preparation of the Title Compounds $\mathbf{5}$}

A solution of 2-methylthio-5-substituted-1,3,4-oxadiazole 4 (3.83 mmol) in glacial acetic acid (10 $\mathrm{mL})$ was treated dropwise at $10{ }^{\circ} \mathrm{C}$ with potassium permanganate $(0.78 \mathrm{~g}, 4.98 \mathrm{mmol})$ as a $5 \%$ aqueous solution over $20 \mathrm{~min}$. The reaction was allowed to proceed for an additional $20 \mathrm{~min}, 40 \%$ strength aqueous sodium hydrogen sulphite solution was subsequently added until the mixture was decolorized, and diluted with $50 \mathrm{~mL}$ of water. The product was filtered and recrystallised from ethanol give the title compounds 5.

2-(Methylsulfonyl)-5-(2-(trifluoromethyl)phenyl)-1,3,4-oxadiazole (5a): yield 86.6\%; white solid; m.p. 102-104 ${ }^{\circ} \mathrm{C}$; ${ }^{1} \mathrm{H}-\mathrm{NMR} \delta: 7.66-7.14$ (m, 4H, ArH), 3.54 (s, 3H, $\left.\mathrm{CH}_{3}\right) ;{ }^{13} \mathrm{C}-\mathrm{NMR} \delta: 162.81,161.81$, 135.43, 135.25, 133.65, 133.29, 112.86, 110.50, 43.12; IR $\left(\mathrm{cm}^{-1}\right)$ v: 2931, 1616, 1558, 1543, 1458, 1338, 1192; Anal. Calcd for $\mathrm{C}_{10} \mathrm{H}_{7} \mathrm{~F}_{3} \mathrm{~N}_{2} \mathrm{O}_{3} \mathrm{~S}$ : C 41.10, H 2.41, N 9.59; found: C 41.46, H 2.02, N 9.88.

2-(Methylsulfonyl)-5-(2,3,4-trifluorophenyl)-1,3,4-oxadiazole (5b): yield 84.3\%; white solid; m.p. 114-115 ${ }^{\circ} \mathrm{C}$; ${ }^{1} \mathrm{H}-\mathrm{NMR} \delta: 7.97-7.32$ (m, 2H, ArH), $3.54\left(\mathrm{~s}, 3 \mathrm{H}, \mathrm{CH}_{3}\right) ;{ }^{13} \mathrm{C}-\mathrm{NMR} \delta: 167.56,161.52$, 161.47, 125.65, 123.74, 121.74, 119.78, 111.23, 43.11; IR $\left(\mathrm{cm}^{-1}\right) v: 3027,2926,1616,1558,1506$, 1350, 1156; Anal. Calcd for $\mathrm{C}_{9} \mathrm{H}_{5} \mathrm{~F}_{3} \mathrm{~N}_{2} \mathrm{O}_{3} \mathrm{~S}$ : C 38.85, H 1.81, N 10.07; found: C 38.56, H 1.51, N 9.82.

2-(Methylsulfonyl)-5-(pyridin-4-yl)-1,3,4-oxadiazole (5c): yield 84.0\%; white solid; m.p. 134-136 ${ }^{\circ} \mathrm{C}$; ${ }^{1} \mathrm{H}-\mathrm{NMR} \delta: 8.41-7.27$ (m, 4H, pyridine-H), 3.54 (s, $\left.3 \mathrm{H}, \mathrm{CH}_{3}\right) ;{ }^{13} \mathrm{C}-\mathrm{NMR} \delta: 167.38,162.45,151.12$, 150.45, 133.12, 121.15, 112.40, 40.32; IR $\left(\mathrm{cm}^{-1}\right) v: 3031,2927,1616,1558,1373$, 1153; Anal. Calcd for $\mathrm{C}_{8} \mathrm{H}_{7} \mathrm{~N}_{3} \mathrm{O}_{3} \mathrm{~S}$ : C 42.66, H 3.13, N 18.66; found: C 41.29, H 2.97, N 19.01.

2-(Methylsulfonyl)-5-(2-fluorophenyl)-1,3,4-oxadiazole (5d): yield 83.7\%; white solid; m.p. 97-99 ${ }^{\circ} \mathrm{C}$; ${ }^{1} \mathrm{H}-\mathrm{NMR} \delta: 8.12-7.29$ (m, 4H, ArH), 3.54 (s, 3H, $\left.\mathrm{CH}_{3}\right) ;{ }^{13} \mathrm{C}-\mathrm{NMR} \delta: 162.30,161.58,159.52,135.34$, 135.27, 130.35, 125.07, 117.50, 117.33, 110.85, 43.08; IR $\left(\mathrm{cm}^{-1}\right)$ v: 3012, 2927, 1616, 1541, 1458, 1340, 1145; Anal. Calcd for $\mathrm{C}_{9} \mathrm{H}_{7} \mathrm{FN}_{2} \mathrm{O}_{3} \mathrm{~S}$ : C 44.63, H 2.91, N 11.56; found: C 44.41, H 2.68, N 11.72.

2-(Methylsulfonyl)-5-(2,6-difluorophenyl)-1,3,4-oxadiazole (5e): yield 80.2\%; white solid; m.p. 131-132 ${ }^{\circ} \mathrm{C}$; ${ }^{1} \mathrm{H}-\mathrm{NMR} \delta: 7.63-7.12(\mathrm{~m}, 3 \mathrm{H}, \mathrm{ArH}) ; 3.53\left(\mathrm{~s}, 3 \mathrm{H}, \mathrm{CH}_{3}\right) ;{ }^{13} \mathrm{C}-\mathrm{NMR} \delta: 162.86,162.02$, 159,94, 159.17, 135.25, 112.89, 112.73, 43.12; IR $\left(\mathrm{cm}^{-1}\right) v: 3022,2931,1629,1587,1477,1352,1153$; Anal. Calcd for $\mathrm{C}_{9} \mathrm{H}_{6} \mathrm{~F}_{2} \mathrm{~N}_{2} \mathrm{O}_{3} \mathrm{~S}$ : C 41.54, H 2.32, N 10.77; found: C 41.41, H 2.01, N 10.48. 
2-(Methylsulfonyl)-5-(2,6-dichlorophenyl)-1,3,4-oxadiazole (5f): yield 84.3\%; white solid; m.p. 126-128 ${ }^{\circ} \mathrm{C}$; ${ }^{1} \mathrm{H}-\mathrm{NMR} \delta: 7.51$ (s, 3H, ArH), 3.55 (s, 3H, $\left.\mathrm{CH}_{3}\right) ;{ }^{13} \mathrm{C}-\mathrm{NMR} \delta: 163.37,162.00,136.65$, 134.00, 128.62, 128.44, 122.53, 43.13; IR $\left(\mathrm{cm}^{-1}\right)$ v: 3028, 2927, 1616, 1587, 1473, 1372, 1153; Anal. Calcd for $\mathrm{C}_{9} \mathrm{H}_{6} \mathrm{Cl}_{2} \mathrm{~N}_{2} \mathrm{O}_{3} \mathrm{~S}: \mathrm{C} 36.88, \mathrm{H}$ 2.06, N 9.56; found: C 36.82, H 1.97, N 9.71.

2-(Methylsulfonyl)-5-(2-methoxyphenyl)-1,3,4-oxadiazole (5g): yield 87.2\%; white solid; m.p. 127-129 ${ }^{\circ} \mathrm{C}$; ${ }^{1} \mathrm{H}-\mathrm{NMR} \delta: 8.02-7.36(\mathrm{~m}, 4 \mathrm{H}, \mathrm{ArH}), 3.54\left(\mathrm{~s}, 3 \mathrm{H}, \mathrm{CH}_{3}\right), 2.74\left(\mathrm{~s}, 3 \mathrm{H}, \mathrm{OCH}_{3}\right) ;{ }^{13} \mathrm{C}-\mathrm{NMR} \delta: 155.79$, 155.32, 132.81, 131.83, 131.36, 129.76, 128.26, 122.49, 43.01, 22.21; IR $\left(\mathrm{m}^{-1}\right)$ v: 3084, 2827, 1635, 1558, 1506, 1458, 1379, 1163; Anal. Calcd for $\mathrm{C}_{10} \mathrm{H}_{10} \mathrm{~N}_{2} \mathrm{O}_{4} \mathrm{~S}$ : C 47.24, H 3.96, N 11.02; found: C 47.19, H 4.28, N 10.96.

2-(Methylsulfonyl)-5-(2-methylphenyl)-1,3,4-oxadiazole (5h): yield 83.4\%; white solid; m.p. 117-119 ${ }^{\circ} \mathrm{C}$; ${ }^{1} \mathrm{H}-\mathrm{NMR} \delta: 8.00-7.08(\mathrm{~m}, 4 \mathrm{H}, \mathrm{ArH}), 3.99\left(\mathrm{~s}, 3 \mathrm{H}, \mathrm{CH}_{3}\right), 3.52\left(\mathrm{~s}, 3 \mathrm{H}, \mathrm{CH}_{3}\right) ;{ }^{13} \mathrm{C}-\mathrm{NMR} \delta: 165.66$, 161.94, 158.56, 134.76, 131.14, 121.01, 112.16, 56.17, 43.04; IR (KBr, $\left.\mathrm{cm}^{-1}\right)$ v: 3010, 2926, 1604, 1533, 1498, 1338, 1155; Anal. Calcd for $\mathrm{C}_{10} \mathrm{H}_{10} \mathrm{~N}_{2} \mathrm{O}_{3} \mathrm{~S}$ : C 50.41, H 4.23, N 11.76; found: C 50.03, $\mathrm{H}$ 4.45, N 11.49 .

2-(Methylsulfonyl)-5-(2-bromophenyl)-1,3,4-oxadiazole (5i): yield 78.8\%; white solid; m.p. 134-135 ${ }^{\circ} \mathrm{C}$; ${ }^{1} \mathrm{H}-\mathrm{NMR} \delta: 8.00-7.46$ (m, 4H, ArH), $3.54\left(\mathrm{~s}, 3 \mathrm{H}, \mathrm{CH}_{3}\right) ;{ }^{13} \mathrm{C}-\mathrm{NMR} \delta: 165.54,162.55,135.02,133.87$, 132.22, 127.96, 123.57, 122.29, 43.07; IR $\left(\mathrm{cm}^{-1}\right)$ v: 3028, 2943, 1615 1557, 1516, 1443, 1373, 1155; Anal. Calcd for $\mathrm{C}_{9} \mathrm{H}_{7} \mathrm{BrN}_{2} \mathrm{O}_{3} \mathrm{~S}$ : C 35.66, H 2.33, N 9.24; found: C 35.87, H 2.44, N 9.61.

2-(Methylsulfonyl)-5-(2-chloro-6-fluorophenyl)-1,3,4-oxadiazole (5j): yield 86.1\%; white solid; m.p. 121-123 ${ }^{\circ} \mathrm{C}$; ${ }^{1} \mathrm{H}-\mathrm{NMR} \delta: 8.89-7.45(\mathrm{~m}, 3 \mathrm{H}, \mathrm{ArH}), 3.543\left(\mathrm{~s}, 3 \mathrm{H}, \mathrm{CH}_{3}\right) ;{ }^{13} \mathrm{C}-\mathrm{NMR} \delta: 165.91,161.87$, 134.71, 134.65, 132.54, 131.23, 127.38, 42.95; IR ( $\left.\mathrm{cm}^{-1}\right) v: 3033,2941,1615,1557,1456,1337,1151$; Anal. Calcd for $\mathrm{C}_{9} \mathrm{H}_{6} \mathrm{ClFN}_{2} \mathrm{O}_{3} \mathrm{~S}$ : C 39.07, H 2.19, N 10.13; found: C 39.02, H 2.29, N 10.11.

\subsection{Antifungal Activities Test}

The antifungal activities were tested against two pathogenic fungi, Fusarium oxysporum and Cytospora mandshurica, by the poison plate technique [29]. Compounds were dissolved in dimethyl sulfoxide $(1 \mathrm{~mL})$ before mixing with potato dextrose agar (PDA, $90 \mathrm{~mL}$ ). The compounds were tested at a concentration of $50 \mu \mathrm{g} / \mathrm{mL}$. All fungi were incubated in PDA at $27 \pm 1{ }^{\circ} \mathrm{C}$ for 4 days to get new mycelium for antifungal assay. Then mycelia dishes of approximately $4 \mathrm{~mm}$ diameter were cut from culture medium, and one of them was picked up with a sterilized inoculation needle and inoculated in the center of PDA plate aseptically. The inoculated plates were incubated at $27 \pm 1{ }^{\circ} \mathrm{C}$ for 5 days. DMSO in sterile distilled water served as negative control, while hymexazol acted as positive control. For each treatment, three replicates were conducted. The radial growth of the fungal colonies was measured and the data were statistically analyzed. The inhibitory effects of the test compounds in vitro on these fungi were calculated by the formula: $I(\%)=[(C-T) /(C-0.4)] \times 100$, where $C$ represents the diameter of fungi growth on untreated PDA, and $T$ represents the diameter of fungi on treated PDA while $I$ means the inhibition rate. 
Some of the title compounds were tested against eight pathogenic fungi namely $F$. oxysporum, C. mandshurica, Phytophthora infestans, Rhizoctonia solani, Thanatephorus cucumeris, Colletotrichum gloeosporioides, Botrytis cinerea and Sclerotinia sclerotiorum at different concentrations of $50,25,12.5,6.25,3.125,0 \mu \mathrm{g} / \mathrm{mL}$. The $\mathrm{EC}_{50}$ (effective dose for $50 \%$ inhibition $\mu \mathrm{g} / \mathrm{mL}$ ) values were estimated statistically by probit analysis with the help of the probit package of the SPSS software using a personal computer. The average $\mathrm{EC}_{50}$ was taken from at least three separate analyses for inhibition of growth using the basic $\mathrm{EC}_{50}$ program version SPSS 11.5.

\section{Conclusions}

In summary, a series of new sulfone compounds containing 1,3,4-oxadiazole moieties and based on the lead compound Ia was designed and synthesized. The title compounds showed promising antifungal activities against some typical fungi often occurring in the Chinese agro-ecosystem, including F. oxysporum, C. mandshurica, R. solani, T. cucumeris, S. sclerotiorum, C. gloeosporioides, $P$. infestans, $B$. cinerea. Among them, some compounds showed superiority over the lead compound Ia, such as compound $\mathbf{5 b}$ with $\mathrm{EC}_{50}$ values ranging from $5.21 \mu \mathrm{g} / \mathrm{mL}$ to $79.2 \mu \mathrm{g} / \mathrm{mL}$, and some, such as compounds $\mathbf{5 b}, \mathbf{5 d}$ and $\mathbf{5 e}$ showed superiority over the commercial fungicide hymexazol. In particular, compounds $\mathbf{5 d}, \mathbf{5 e}, \mathbf{5 f}$, and $\mathbf{5 i}$ showed prominent activity against $B$. cinerea, with $\mathrm{EC}_{50}$ values of $5.21 \mu \mathrm{g} / \mathrm{mL}, 8.25 \mu \mathrm{g} / \mathrm{mL}, 8.03 \mu \mathrm{g} / \mathrm{mL}$, and $21.00 \mu \mathrm{g} / \mathrm{mL}$, respectively. These results demonstrated that some compounds such as $\mathbf{5 d}$ can be used as possible lead compounds for the development of potential agrochemicals.

\section{Acknowledgments}

We are grateful to the National Natural Science Foundation of China (Nos. 20962005, 20762002), the National Key Technology R\&D Program (Nos. 2011BAE06B05-6, 2011BAE06B02-14, 2011BAE06B02-09), S\&T Program of Guizhou Province (No. [2011]3016) for supporting the project.

\section{Conflict of Interest}

The authors declare no conflict of interest.

\section{References and Notes}

1. Russell, P.E. A century of fungicide evolution. J. Agric. Sci. 2005, 143, 11-25.

2. Copping, L.G.; Menn, J.J. Biopesticides: A review of their action, applications and efficacy. Pest. Manag. Sci. 2000, 56, 651-767.

3. Fitzjohn, S.; Robinson, M.P. Benzoxazole and benzothiazole derivatives. WO 9406783, 1994 (Chem. Abst. 1994, 121, 9394f).

4. Hiromichi, I.; Masakazu, T.; Ten, U.; Seiichi, K. Preparation of disulfonylthiadiazoles and their use as agrochemical microbicides. JP 94116252, 1994 (Chem. Abst. 1994, 121, 127847).

5. Plant, A.; Boehmer, J.E.; Black, J.; Sparks, T.D. Isoxazoline derivatives and their preparation, herbicidal composition, and use as herbicides to control weeds or plant growth inhibition. WO 2006024820, 2006 (Chem. Abst. 2006, 144, 274262v). 
6. Gong, P.; Chai, H.F.; Zhao, Y.F.; Zhao, C.S. Synthesis and in vitro anti-hepatitis B virus activities of some ethyl 5-hydroxy-1H-indole-3-carboxylates. Bioorg. Med. Chem. 2006, 14, 2552-2558.

7. Tai, X.S.; Yin, X.H.; Tan, M.Y. Crystal structure and antitumor activity of tri[2-[N-(4'-methylbenzylsulfonyl)amino]ethyl]-amine. Chin. J. Struct. Chem. 2003, 22, 411-414.

8. Fang, S.H.; Padmavathi, V.; Rao, Y.K.; Subbaiah, D.R.C.; Thriveni, P.; Geethangili, M.; Padaja, A.; Tzeng, Y.M. Biological evaluation of sulfone derivatives as anti-inflammatory and tumor cells growth inhibitory agents. Int. Immunopharmacol. 2006, 6, 1699-1705.

9. Vedula, M.S.; Pulipaka, A.B.; Venna, C.; Chintakunta, V.K.; Jinnapally, S.; Kattuboina, V.A.; Vallakati, R.K.; Basetti, V.; Akella, V.; Rajgopai, S.; et al. New styryl sulfones as anticancer agents. Eur. J. Med. Chem. 2003, 38, 811-824.

10. Silvestri, R.; Artico, M.; Regina, G.L. Anti-HIV-1 activity of pyrryl aryl sulfone (PAS) derivatives: Synthesis and SAR studies of novel esters and amides at the position 2 of the pyrrole nucleus. Farmaco 2004, 59, 201-210.

11. Talath, S.; Gadad, A.K. Synthesis, antibacterial and anti-tubercular activities of some 7-[4-(5amino-[1,3,4]thiadiazole-2-sulfonyl)-piperazin-1-yl] fluoroquinolonic derivatives. Eur. J. Med. Chem. 2006, 41, 918-924.

12. Diehr, H.J.; Marhold, A.; Brandes, W.; Hanssler, G. Preparation of 2,5-bis (alkylsulfonyl)-1,3,4thiadiazoles as agrochemical fungicides. DE 3838432, 1990 (Chem. Abst. 1990, 113, 191364f).

13. Assmann, L.; Stenzel, K.; Erdelen, C.; Kugler, M.; Wachtler, P. Nitrophenyl sulfonyl imidazoles and use thereof for controlling vegetable and animal pests. US 20020094936 A1, 2002 (Chem. Abst. 2002, 130, 125074).

14. Yuan, D.K.; Li, Z.M.; Zhao, W.G.; Chen, H.S. Synthesis and bioactivity of 2-substituted amino-5pyrazolyl-1,3,4-oxadiazoles. Chin. J. Appl. Chem. 2003, 20, 624-628.

15. Ohshima, T.; Komyojia, T.; Mitani, S. Development of a novel fungicide, eyazofamid. J. Pestic. Sci. 2004, 29, 136-138.

16. Komyji, N.; Terumasa, K.; Kazumi, S.; Keiichiro, I. Imidazole compounds and biocidal compositions comprising the same. EP 0298196A1, 1989 (Chem. Abst. 1989, 110, 192824).

17. Liu, Z.; Yang, G.; Qin, X. Syntheses and biological activities of novel diheterocyclic compounds

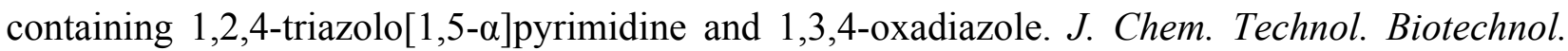
2001, 76, 1154-1158.

18. Jiang, L.; Tan, Y.; Zhu, X.; Wang, Z.; Zuo, Y.; Chen, Q.; Xi, Z.; Yang, G.F. Design, synthesis, and 3D-QSAR analysis of novel 1,3,4-oxadiazol-2(3H)-ones as protoporphyrinogen oxidase inhibitors. J. Agric. Food Chem. 2010, 58, 2643-2651.

19. Qian, X.; Zhang, R. Syntheses and insecticidal activities of novel 2,5-disubstituted-1,3,4oxadiazoles. J. Chem. Tech. Biotechnol. 1996, 67, 124-130.

20. Cao, S.; Qian, X.; Song, G.; Chai, B.; Jiang, Z. Synthesis and antifeedant activity of new oxadiazolyl 3(2H)-pyridazinones, J. Agric. Food Chem. 2003, 51, 152-155.

21. Keshari, K.J.; Abdul, S.; Yatendra, K.; Mohd, S.; Ratan, L.K.; Jainendra, J.; Vikash, K.; Priyanka, S. Design, synthesis and biological evaluation of 1,3,4-oxadiazole derivatives. Eur. J. Med. Chem. 2010, 45, 4963-4967. 
22. Chen, C.J.; Song, B.A.; Yang, S.; Xu, G.F.; Bhadury, P.S.; Jin, L.H.; Hu, D.Y.; Li, Q.Z.; Liu, F.; Xue, W.; et al. Synthesis and antifungal activities of 5-(3,4,5-trimethoxyphenyl)-2-sulfonyl-1,3,4thiadiazole and 5-(3,4,5-trimethoxyphenyl)-2-sulfonyl-1,3,4-oxadiazole derivatives. Bioorg. Med. Chem. 2007, 15, 3981-3989.

23. Liu, F.; Luo, X.Q.; Song, B.A.; Bhadury, P.S.; Yang, S.; Jin, L.H.; Xue, W.; Hu, D.Y. Synthesis and antifungal activity of novel sulfoxide derivatives containing trimethoxyphenyl substituted 1,3,4-thiadiazole and 1,3,4-oxadiazole moiety. Bioorg. Med. Chem. 2008, 16, 3632-3640.

24. Pees, B.; Paul, J.M.; Oget, N.; Sindt, M.; Mieloszynski, J.L. Synthesis of fluoro-substituted monomers bearing a functionalised lateral chain: Part 2. Preparation of sulfoxides and sulfones containing monomers. J. Fluorine Chem. 2003, 124, 139-146.

25. Yamazaki, S. Selective synthesis of sulfones and sulfoxides by methytrioxorhenium catalyzed oxidation of sulfides with hydrogen peroxide. Bull. Chem. Soc. Jpn. 1996, 69, 2955-2959.

26. Confalone, P.N.; Woodward, R.B. A novel synthesis of peptides based on the photochemistry of 5-azido-1,3,4-oxadiazoles. J. Am. Chem. Soc. 1983, 105, 902-906.

27. Song, B.A.; Chen, C.J.; Yang, S.; Jin, L.H.; Xue, W.; Zhang, S.M.; Zou, Z.H.; Hu, D.Y. Synthesis, structure and antitumor activity of 2-alkylthio-5-(3,4,5-trimethoxyphenyl)-1,3,4-thiadiazole compounds. Acta Chim. Sinica 2005, 63, 1720-1726.

28. Chen, C.J.; Song, B.A.; Yang, S.; Xu, G.F.; Bhadury, P.S.; Jin, L.H.; Hu, D.Y.; Li, Q.Z.; Liu, F.; Xue, W.; et al. Synthesis and antifungal activities of 5-(3,4,5-trimethoxyphenyl)- 2-sulfonyl-1,3,4thiadiazole and 5-(3,4,5-trimethoxyphenyl)-2-sulfonyl-1,3,4-oxadiazole derivatives. Bioorg. Med. Chem. 2007, 15, 3981-3989.

29. Tarun, K.C.; Prem, D.J. Antifungal activity of 4-methyl-6-alkyl-2H-pyran-2-ones. J. Agric. Food Chem. 2006, 54, 2129-2133.

Sample Availability: Samples of the compounds are available from the authors.

(C) 2011 by the authors; licensee MDPI, Basel, Switzerland. This article is an open access article distributed under the terms and conditions of the Creative Commons Attribution license (http://creativecommons.org/licenses/by/3.0/). 